\title{
Lutetium Lu 177-DOTA-di-HSG Peptide IMP-288
}

National Cancer Institute

\section{Source}

National Cancer Institute. Lutetium Lu 177-DOTA-di-HSG Peptide IMP-288. NCI

Thesaurus. Code C82354.

A radiolabeled divalent histamine-succinyl-glycine (HSG) hapten-peptide linked with the macrocyclic chelator 1,4,7,10-tetraazacyclododecane-1,4,7,10-tetraacetic acid (DOT A) to the radionuclide lutetium (Lu) 177. After pretargeting with a bispecific monoclonal antibody (BiMoAB) directed against both a tumor associated antigen (TAA) and the HSG hapten-peptide, the HSG portion of administered Lu-177-labeled di-HSG-DOTA peptide IMP-288 binds the anti-HSG portion of the BiMoAB; Lu-177 radioisotopic activity localized to tumor cells bearing the TAA can then be visualized scintig raphically. 